\title{
L'aqueduc de Carthage
}

E. Clamagirand, S. Rais, J. Chahed, R. Guefrej, L. Smaoui (*)

Ecole nationale d'ingénieurs de Tunis

Campus universitaire, B.P. 37, 1002 Tunis - Tunisie

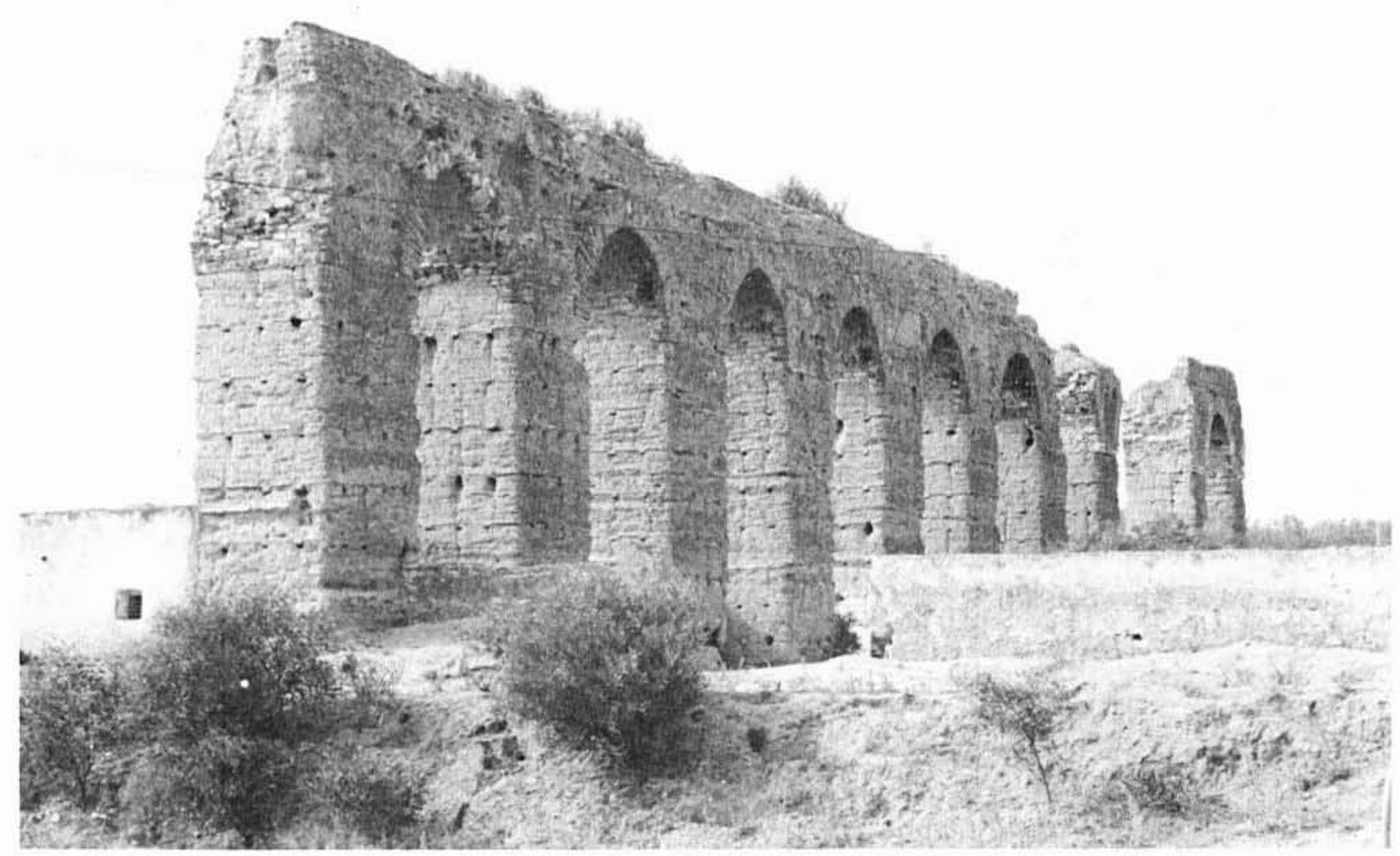

The Carthage Aqueduct

The hydraulic study of the Carthage Aqueduct has been undertaken. After determining the Strickler coefficient by a numeric method and an experimental method and the reconstruction of a length profile, we calculated the capacity of the Aqueduct. The town's consummation of water at the time of the romans, deduced from these results, was less than that of Rome and Pompei at the same period. But all show a great amount of waste, confirmed by the existence of highly developed sewerage networks. 


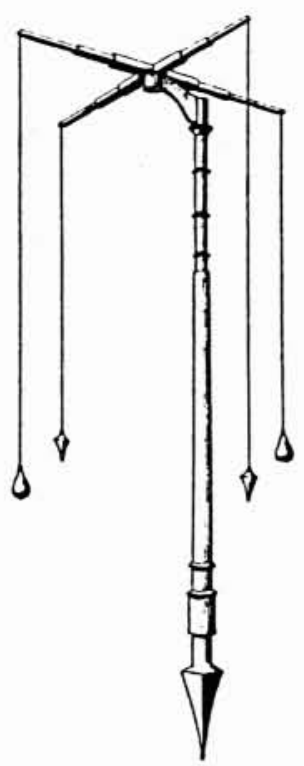

b) Groma

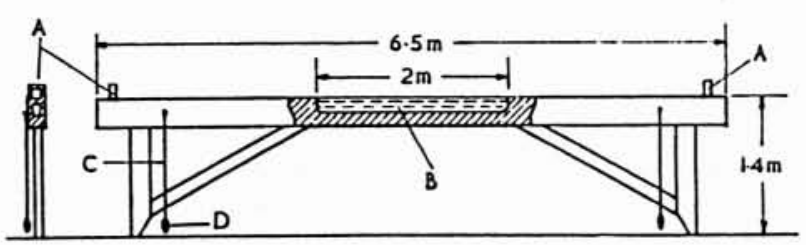

a) Chorobate

\section{Introduction}

L'aqueduc de Carthage, vestige romain de plus de 18 siècles d'âge, parcourt les environs de Tunis sur de longs kilomètres. Il a été utilisẻ au cours des siècles et l'est encore, de nos jours, sur de très longs tronçons par la Société nationale d'exploitation des eaux (SONEDE). Sa beauté, son état de conservation n'ont pas manqué de susciter de nombreuses questions quant au degré de connaissances techniques, hydrauliques et architecturales des Romains.

Ces questions sont à l'origine de ce travail dont le but est, à partir de documents historiques, cartographiques, de mesures expérimentales et d'études numériques, d'arriver à une meilleure connaissance de l'ouvrage, en définissant notamment sa capacité de transport.

\section{Historique}

Le développement des aqueducs dans l'Empire romain a suivi l'expansion urbaine et les exigences humaines, agricoles ou industrielles. Ces demandes en eau les ont poussé à rechercher cette ressource de plus en plus loin de leurs lieux d'habitation et à introduire pour la véhiculer des techniques alors inconnues dans l'Antiquité: arcades, siphons, mesures des pentes.

Parallèlement, un droit de l'eau, réglant aussi bien les aspects techniques que juridiques, s'est développé, comme en témoignent les ouvrages de Pline ou Vitruve (Traité de l'eau).
Le captage de l'eau a diverses origines : rivières et lacs pour les eaux agricoles, sources pour les eaux domestiques. Dans ce dernier cas, l'eau est captée par des galeries drainantes débouchant dans un réservoir d'où part l'aqueduc.

L'aqueduc lui-même est un ouvrage de section généralement rectangulaire, surmonté d'une voûte et dans lequel l'eau s'écoule gravitairement. Les dimensions de la section sont susceptibles de varier en fonction des variations du débit ou de la pente.

La pente des aqueducs est mesurée au moyen de deux appareils: le chorobate (niveau) (fig. la) et la groma (appareil de visée) (fig. $1 b$ ). La faible précision de ces appareils explique la présence de chutes brusques ou contre-pentes sur le profil en long des aqueducs. La pente est faible, quand le relief le permet, afin de ne pas porter préjudice à l'état de l'ouvrage et c'est pourquoi celui-ci serpente souvent, contournant les obstacles. Des valeurs maximales sont conseillées par différents auteurs : $0,2 \%$ (Pline) ; $5 \%$ (Vitruve), 9,7\%o (Palladius). Leur dispersion traduit chez les romains une lacune dans leurs connaissances de l'hydraulique.

L'étanchéité de l'ouvrage est assurée par un mortier, caractéristique de l'époque romaine, constitué de chaux, de sable et de briques concassées.

Mais les connaissances hydrauliques des ingénieurs romains se manifestent le mieux dans les nombreux ouvrages annexes aux aqueducs : les siphons ou les arcades pour franchir les dépressions (l'exemple le plus célèbre est le Pont du Gard en France), les filtres et bassins de décantation pour la qualité de l'eau, les regards fréquents pour le contrôle de l'ouvrage. 


\begin{tabular}{|c|c|c|c|c|c|}
\hline Aqueduc & Situation & Longueur $(\mathrm{km})$ & $\begin{array}{l}\text { Pente moyenne } \\
\% \text { \% }\end{array}$ & $\begin{array}{c}\text { Débit estimé } \\
\mathrm{m}^{3} / \text { jour }\end{array}$ & Date \\
\hline Carthage & $\begin{array}{c}\text { Carthage } \\
\text { Tunisie }\end{array}$ & 132 & 3 & 32000 & $+160 ?$ \\
\hline Aqua Marcia & $\begin{array}{l}\text { Rome } \\
\text { Italie }\end{array}$ & 91 & 1,54 & 188000 & -144 \\
\hline Brevenne & $\begin{array}{l}\text { Lyon } \\
\quad \text { France }\end{array}$ & 66 & 5,3 & 28000 & +0 à 50 \\
\hline Cherchell & $\begin{array}{r}\text { Cherchell } \\
\text { Algérie }\end{array}$ & 45 & 2 & & \\
\hline Craponne & $\begin{array}{l}\text { Lyon } \\
\quad \text { France }\end{array}$ & 25 & 16,8 & 13000 & -12 \\
\hline Aqua Appia & $\begin{array}{l}\text { Rome } \\
\text { Italie }\end{array}$ & 16 & $\min =0.5$ & 73000 & -312 \\
\hline
\end{tabular}

Le débouché de l'aqueduc dans la ville doit répondre à deux problèmes:

- briser l'excédent éventuel de charge, afin de ne pas détériorer les réseaux urbains. Ainsi, à Pompéi, un château d'eau permettait d'abaisser les pressions d'eau de 1,8 bar à 0,6 bar ;

- répartir l'eau entre les différents utilisateurs en respectant les priorités (fontaines publiques, thermes, particuliers). L'ouvrage de répartition ou Castellum Divisorium est un bassin muni de vannes qui permettaient de réguler la distribution.

Les principaux aqueducs de l'Empire Romain, connus de nos jours, sont énumérés dans le tableau ci-dessus, avec leurs caractéristiques.

\section{L'aqueduc de Carthage}

La première colonie romaine s'installe à Carthage en 44 av. J.-C. autour du petit village de La Malga (fig. 2). En 144 ap. J.-C., se dresse une cité sur l'actuelle colline de Byrsa. La ville s'étendra très vite et Carthage deviendra la deuxième ville de l'Empire Romain après Rome. La population des deux villes au début du deuxième siècle est controversée : sur la base d'une population d'un million d'habitants à Rome, Lézine (1969) estime celle de Carthage à 300000 hab. ; mais des estimations plus récentes (d'après G. Ch. Picard, in Lézine) affectent 350000 hab. à Rome et 100000 à Carthage.

Traditionnellement, la ville était alimentée par des citernes (citernes de La Malga et de Borj Djedid) et des fontaines. La construction de l'aqueduc remonte au milieu du deuxième siècle ap. J.-C., relativement tard dans l'histoire de la ville. Il prend sa source à Zaghouan (fig. 2), au lieu-dit "Sanctuaire des eaux ». Les eaux de source y sont drainées par deux galeries et se déversent dans un bassin double (fig. 3, page suiv.) par des passages laissés libres entre les joints. De là, l'eau s'écoule directement dans l'aqueduc par une sortie rectangulaire. Une dizaine d'années après l'exploitation de ces sources, une branche véhiculant les eaux du Nymphée d'Aîn Djouggar fut construite, afin de constituer un débit d'apport supplémentaire.

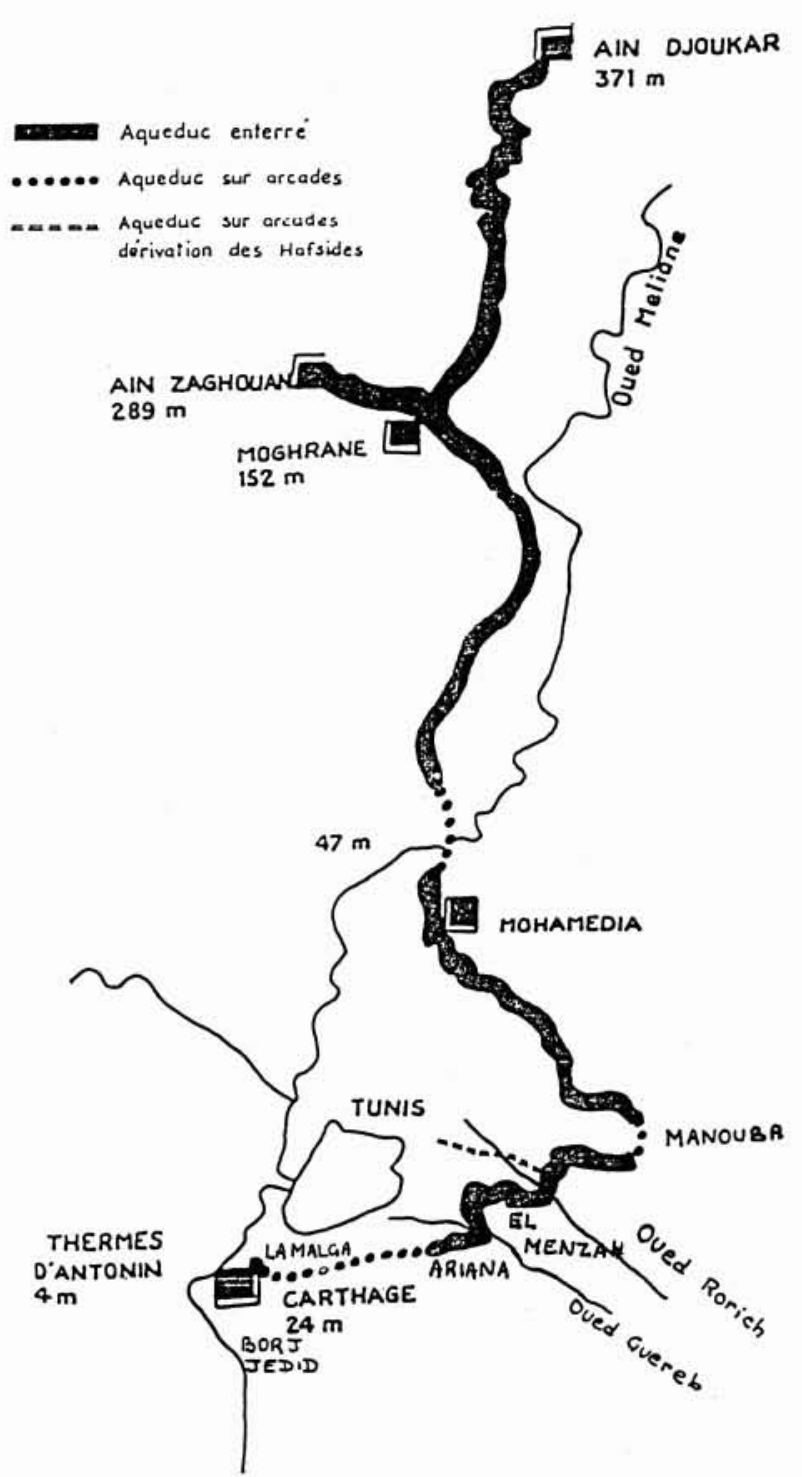

2. Plan de situation (éch. : $1 / 310000$ ). 


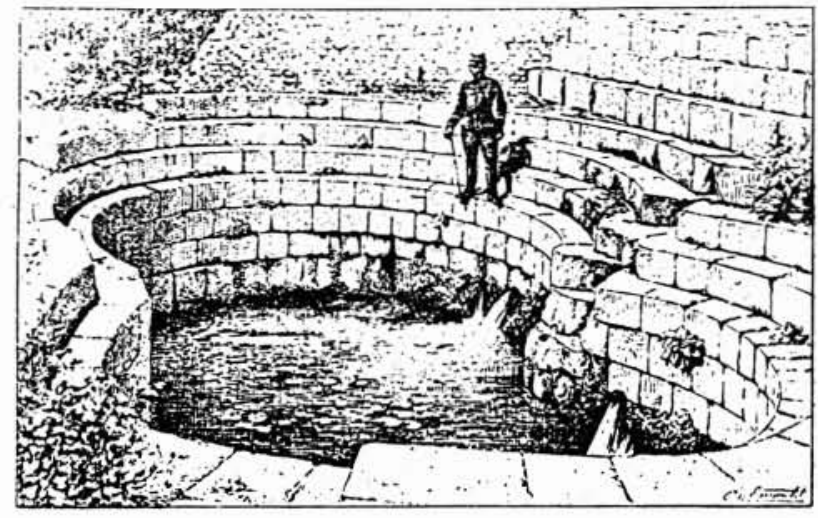

3. Bassin.double du Nymphée de Zaghouan (d'après Rakob, 1974).

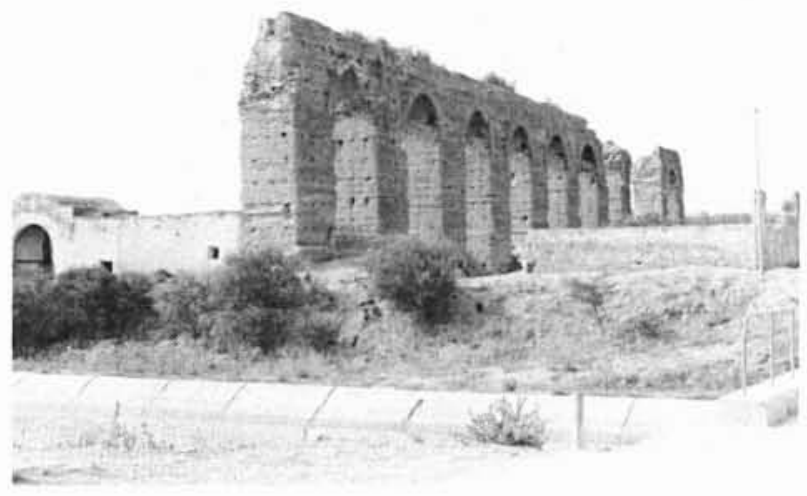

4. Traversée de l'oued Miliane.

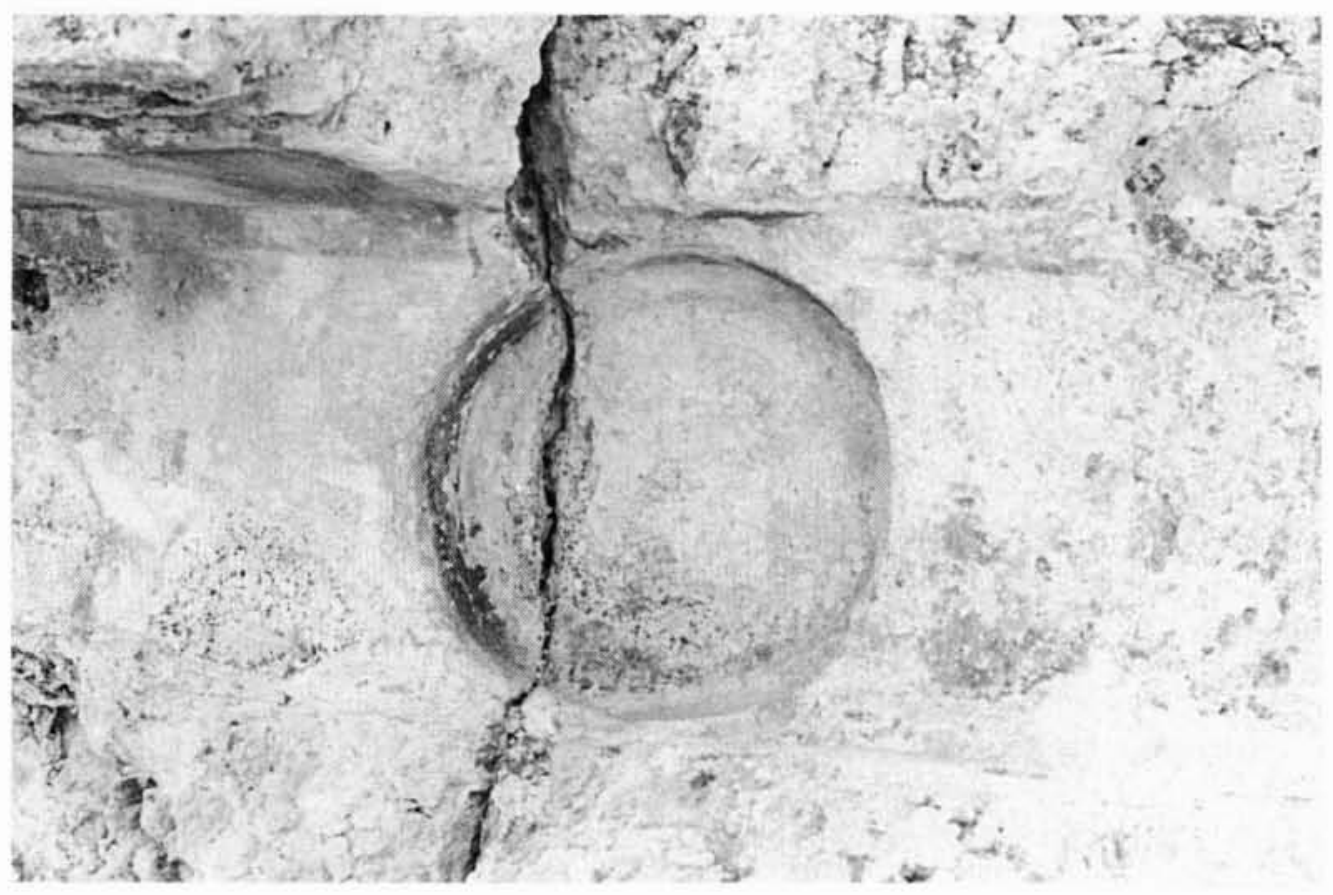

5. Piège à sédiments.

La longueur totale de l'aqueduc (deux branches comprises) est de $132 \mathrm{~km}$ dont 17 aériens dans la plaine de l'oued Miliane (arcades à doubles étages de $32 \mathrm{~m}$ de hauteur) et dans la dépression de Manouba (fig. 4). C'est l'aqueduc le plus long parmi les aqueducs connus du monde romain (voir le tableau page précédente).

Sa pente varie entre $0,1 \%$ et $9,58 \%$ en région montagneuse (entre Zaghouan et Moghrane). Dans les régions à faible pente (à l'aval), des fosses circulaires dans le radier tiennent lieu de pièges à sédiments (fig. 5).

La section de l'aqueduc est trapézoïdale. Les fruits des parois sont toutefois très faibles et les dimensions moyennes de la section sont de l'ordre de $1,60 \mathrm{~m} \times 0,80 \mathrm{~m}$, avec parfois une cunette pour améliorer l'écoulement dans certaines parties de l'ouvrage en cas de faibles débits (Fig. 6 et 7 ). 


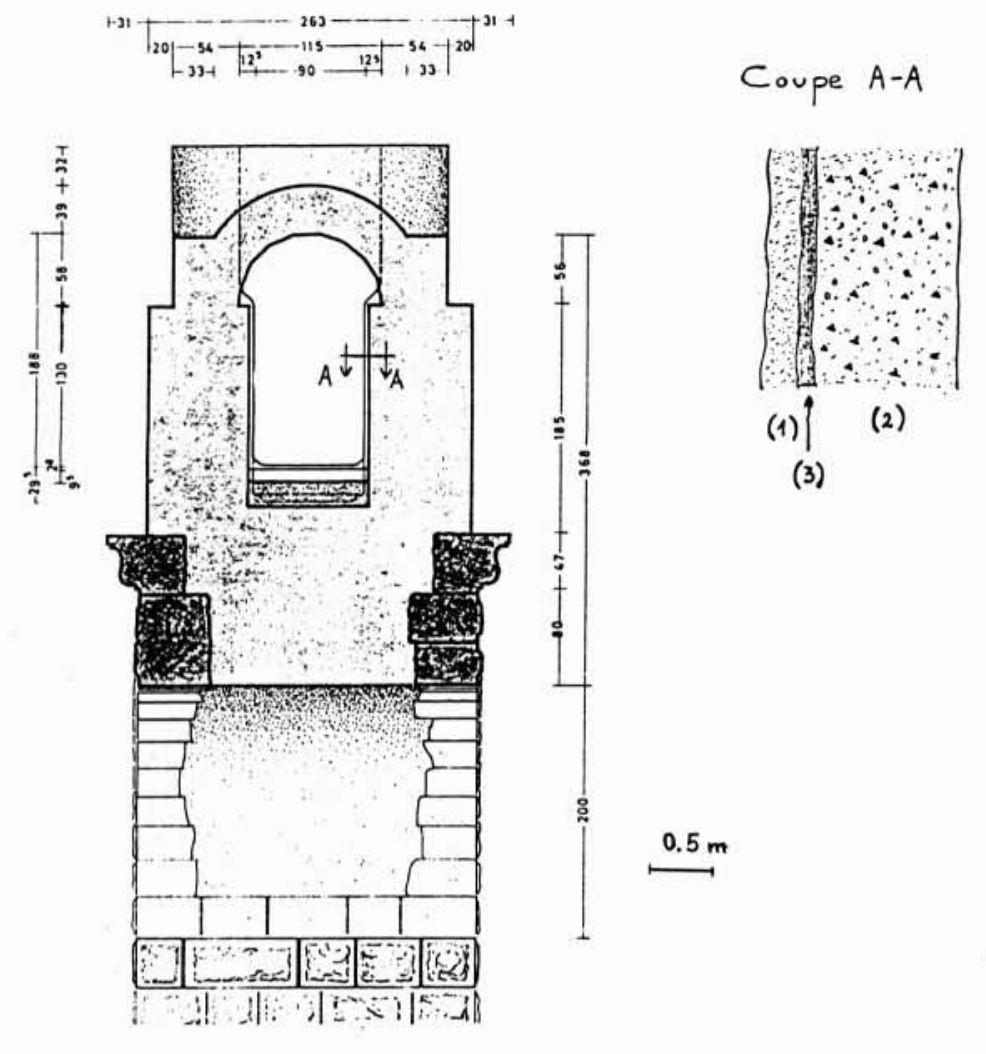

6. Section de l'aqueduc (d'après Rakob, 1974).

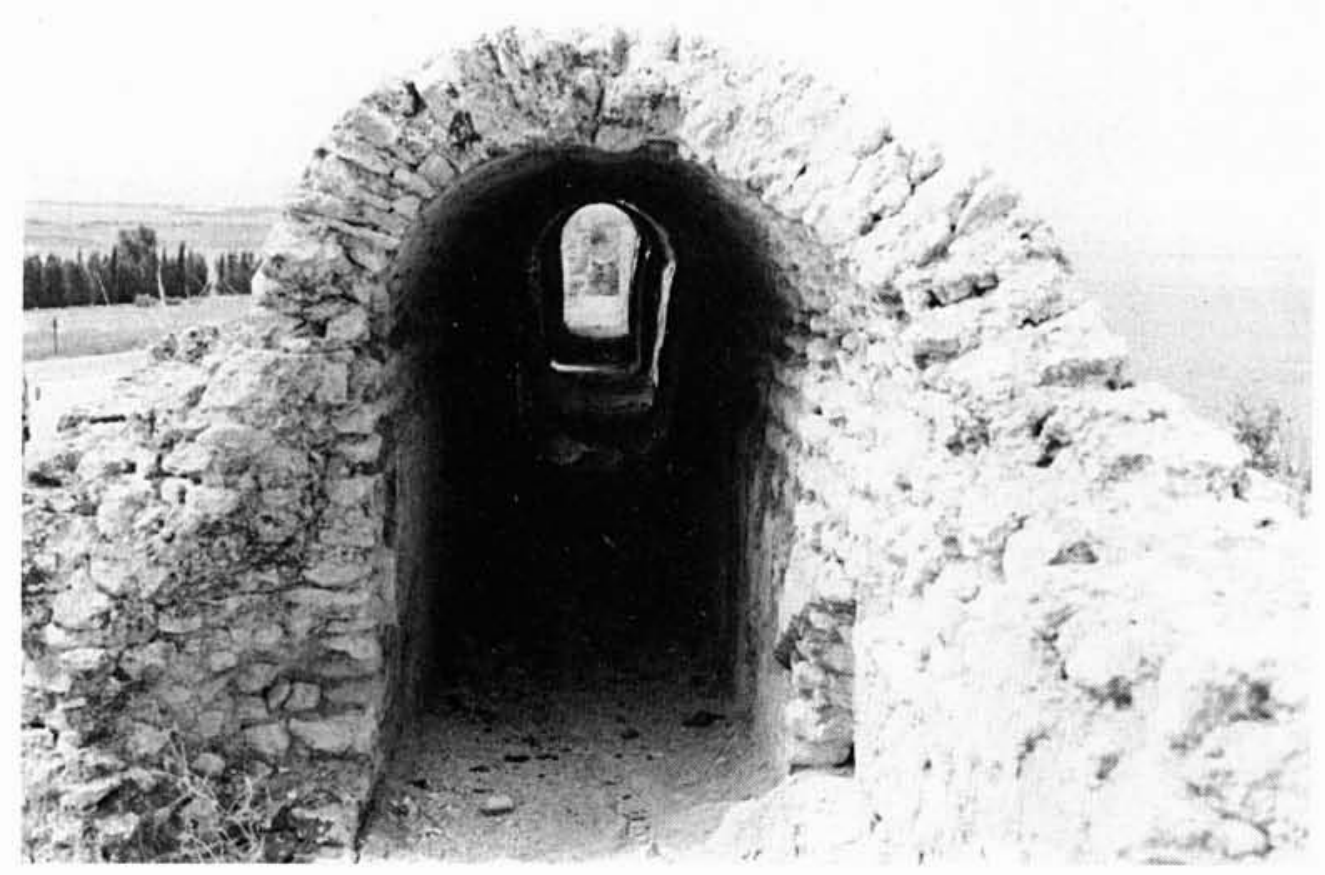

7. Section de l'aqueduc à la Mohammedia. 
Enfin, l'ouvrage est doté de regards à intervalles réguliers de $200 \mathrm{~m}$ et de puits circulaires en maçonnerie de diamètre égal à la largeur de l'aqueduc.

Le débouché de l'aqueduc dans la ville est inconnu. L'ouvrage se divisait certainement en plusieurs branches. A l'aval, il est visible seulement au niveau des citernes de la Malga. A partir de là, plusieurs hypothèses sont envisagées :

- une branche de l'aqueduc passant le long des citernes de la Malga alimentait celles-ci par déversement,

- une autre branche, à angle droit de la première, prend la direction des citernes de Borj Djedid et des thermes d'Antonin. Peut-être rejoignait-elle aussi un castellum divisorium, dont on n'a pas retrouvé de traces si ce n'est sur des gravures (Musée du Bardo, Tunis) ou des gobelins (Casa Real, Séville).

L'aqueduc fut restauré plusieurs fois au cours de son histoire : en 534 (administration Bélisaire), sous les Fatimides, en 1850 (Bey Mohamed) et à l'époque coloniale où les arcades ont été remplacées par des siphons.

De nos jours, la SONEDE exploite encore les deux sources, qui ont été équipées de pompes et utilise l'ouvrage sur $60 \mathrm{~km}$ entre Zaghouan et Bou Naouara.

\section{Estimation du frottement}

Afin d'évaluer le débit de l'aqueduc, il est nécessaire de déterminer le frottement sur le fond et les parois, représenté par la formule de Manning-Strickler. Le coefficient K de Manning-Strickler a été estimé de deux façons :

\subsection{Méthode numérique}

Sur la base de mesures de débits et tirants d'eau sur les tronçons actuellement en service, nous avons effectué un calage du coefficient de Strickler, à partir d'un calcul de courbes de remous dans l'aqueduc. Le tronçon étudié est de $16,345 \mathrm{~km}$ de longueur entre Jebel Oust et la tête amont du siphon de l'oued Miliane. Les mesures de tirants d'eau ont été effectuées par la SONEDE en des points distants de 70 à $1200 \mathrm{~m}$. Ces mesures sont relatives à deux débits $\left(9000 \mathrm{~m}^{3} / \mathrm{j}\right.$ et $\left.18000 \mathrm{~m}^{3} / \mathrm{j}\right)$ et datent de 1967 . Dans ces deux cas, le régime est fluvial et le calcul commence de l'aval. Malgré la dispersion des résultats, due à des dépôts qui augmentent localement le frottement, le coefficient de Strickler moyen a été estimé à $\mathrm{K}=30$. Nous considérons que, compte-tenu des dépôts, cette valeur constituera une limite inférieure du Strickler à l'époque romaine.

\subsection{Méthode expérimentale}

Le coefficient $\mathrm{K}$ de frottement dépend essentiellement de la rugosité de la section mouillée. Afin de la déterminer, l'état initial à l'époque romaine de cette section doit être reconstitué après plusieurs siècles de dégradation et d'éventuels changements.
A la suite d'inspections effectuées sur les différents lieux des ruines et des tronçons de l'aqueduc, il est apparu que les matériaux du radier et des parois sont constitués dans certains lieux (comme la Mohamedia) de deux couches (voir la fig. 6, coupe A-A) :

(1) l'une supérieure, d'épaisseur variable mais faible, est constituée de matériaux fins et assimilée à première vue à un dépôt ;

(2) la couche en dessous, épaisse et plus rugueuse, commune à toutes les sections se présente comme un mortier (constituée principalement de quartz, de chaux et de morceaux de terre cuite).

Sur d'autres tronçons de l'aqueduc (à la Malga et à la Soukra, fig. 2) on a noté la présence d'une troisième couche (3) lisse et grisâtre intercalée entre les deux précédentes. Deux hypothèses ont été faites sur la nature de cette couche :

- hypothèse a) : on assimile cette couche à un revêtement, - hypothèse b) : on assimile cette couche à un dépôt.

Dans les deux cas, la couche supérieure est un dépôt.

Dans le but de trancher entre ces hypothèses et vu l'état médiocre des échantillons qui sont dans la plupart du temps dégradés et friables, nous avons effectué sur ces échantillons des analyses chimiques et granulométriques (par voie humide).

Des essais en laboratoire relatifs à la détermination de la porosité et de l'imperméabilité ont été infructueux. II faudrait essayer dans le futur de les mesurer sur site.

Les analyses chimiques ont pour but de caractériser les différentes couches des sections (trouvées dans les différents sites) et de confirmer ou d'infirmer l'hypothèse de leur homogénéité.

Ces essais ont consisté à doser dans ces couches le calcium, le carbonate de calcium, la silice et le magnésium et à utiliser quelques-unes de leurs propriétés qui permettent, en comparant la composition de ces couches, d'aider à trancher entre les deux hypothèses proposées.

Les résultats des essais ont montré que l'hypothèse (a) est confirmée, donc :

- pour les tronçons de la Mohammedia, l'eau coulait directement sur le mortier et la couche (1) correspondante est un dépôt,

- pour le cas de la Soukra et de la Malga, l'eau coulait sur un revêtement.

En plus des analyses et toujours dans le même but, les échantillons ont été passés à la diffraction X. Les résultats ont bien confirmé ceux des analyses chimiques.

Enfin en vue de déterminer les diamètres représentatifs des granulats pour les différentes couches, nous avons procédé après concassage à l'analyse granulométrique des échantillons ; ces analyses étaient assez délicates du fait que l'on voulait reconstituer la granulométrie d'un matériau mis en place et en contact avec des abrasions atmosphériques depuis plus de dix-huit siècles. Il ressort des courbes une certaine homogénéité : le diamètre représentatif a été déterminé à partir d'une courbe moyenne. 
La valeur du coefficient $\mathrm{K}$ pour le mortier a alors été déduite de la formule de Strickler reliant $\mathrm{K}$ au diamètre $\mathrm{D}_{90}$ du mélange (Larras, 1972). Elle est dans une gamme proche de celle estimée numériquement.

Dans le cas où il existe un revêtement, celui-ci a été assimilé à un ciment rugueux; le coefficient de Strickler serait de 50 selon Chow (1959).

Ainsi, les romains n'auraient prévu un revêtement que dans la partie aval. Ceci se justifie hydrauliquement par la topographie du terrain : le handicap des faibles pentes dans les dépressions de La Soukra et de La Malga a conduit les concepteurs à améliorer les conditions d'écoulement et d'imperméabilité en améliorant la rugosité par un revêtement.

\section{Reconstitution du profil en long}

Les différents traitements hydrauliques de l'aqueduc nécessitent une caractérisation géométrique du canal (section, pente, ouvrages...). Ceci nous a amenés à considérer le tracé en plan et le profil en long du monument sur la base des ruines et documents disponibles.

La partie amont de l'aqueduc, du fait des successives réhabilitations et aménagements qu'elle a subis tout au long de l'histoire, demeure encore en bon état et continue même à être utilisée par les services tunisiens de distribution d'eau potable. Dans la partie aval par contre, les ruines de l'aqueduc sont, la plupart du temps en mauvais état ou complètement détruites à tel point qu'on perd même les traces du monument dans plusieurs endroits et sur de longues distances.

L'ensemble des documents disponibles fait ressortir une connaissance quasi totale du profil en long de l'aqueduc dans sa partie amont longue de $60 \mathrm{~km}$ environ et qui s’étend de Zaghouan à Bou Naouara. Le profil en long de ce tronçon, établi au début du siècle donne tous les détails sur les ouvrages, sections...

Les sections du canal, dont la forme moyenne est donnée par la figure $5 a$, ne présentent pas de variations notables sur toute la partie amont. Ceci nous a amenés à adopter cette section moyenne dans toutes les parties où il nous a été possible de la reconstituer.

Sur la partie aval de l'aqueduc qui lie Bou Naouara à Carthage, l'ensemble des documents disponibles ne présentent qu'un tracé incomplet. Ces documents sont:

- carte échelle 1/100 000 établie par P. Colin (1862),

- carte échelle 1/100 000 établie par F. Rakob (1974),

- carte d'état major échelle 1/200 000 (1928),

- carte d'état major échelle 1/50 000 (1921),

- plan de restitution échelle 1/5000 (1941-1982-1984).

La carte établie par François Rakob n'est qu'une reproduction de l'ancien plan réalisé par Pierre Colin. Le tracé de l'aqueduc depuis les sources jusqu'à la Cité de Carthage ne figure que sur ces deux plans qui, du point de vue topographique sont inexploitables.
L'idée est donc de partir de ces documents de base et des ruines encore existantes du monument pour compléter le plus fidèlement possible les tracés en plan incomplets sur les autres plans. Le but serait de porter la totalité du tracé en plan sur le plan de restitution à l'échelle $1 / 5000$, à partir duquel on connait assez précisément les cotes du sol.

La reconstitution du profil en long est alors possible en mesurant sur toutes les parties de l'aqueduc où c'est possible les cotes du fond du canal par rapport au fond.

Cette démarche, bien qu'elle nous ait permis de compléter la majeure partie du tracé, n'a pas été concluante dans certains passages: c'est le cas de presque toute la zone urbaine du Grand Tunis (El Menzah, l'Ariana, fig. 3), où, par suite de l'urbanisation des dernières décennies, les traces de l'aqueduc ont presque entièrement disparu. Dans les meilleurs des cas, des enquêtes in situ, auprès de la population, nous ont permis de retrouver quelques vestiges, notamment au niveau des oueds où le monument passait sur des arches (oued Guéreb, fig. 2). Pour la région de l'Ariana où les traces sont rares, des hypothèses portant sur le tracé et sur le profil de l'aqueduc ont été formulées compte tenu des tronçons existant à l'amont et l'aval, de la topographie du terrain et des techniques habituelles aux romains (contournement des collines, pentes faibles). Ces hypothèses, confrontées entre elles, ont été relativement validées par des considérations hydrauliques et des témoignages: celles qui s'avèrent les plus plausibles ont été retenues.

Le profil en long ainsi reconstitué présente des pentes très faibles (généralement inférieures à $0,04 \%$ ) et il est clair que les concepteurs du monument ont recherché un tracé en plan très compliqué mais qui présente l'avantage d'utiliser au mieux les moindres déclivités du terrain naturel. C'est ce qui nous a amené à penser que le tracé en plan et le profil établis semblent être corrects.

En effet, une erreur sur les cotes ou sur le tracé d'un tronçon, même relativement faible, se traduirait vraisemblablement par des contrepentes inadmissibles sur les autres.

\section{Etude hydraulique - Estimation des débits}

La valeur du débit maximal véhiculé par l'aqueduc est controversée: les archéologues F. Rakob (1979) et $\mathrm{Ph}$. Caillat (1873) l'ont estimée à $32000 \mathrm{~m}^{3} / \mathrm{j}$, tandis que la SONEDE donne une valeur maximale de $20000 \mathrm{~m}^{3} / \mathrm{j}$, voire $11000 \mathrm{~m}^{3} / \mathrm{j}$ sur certains tronçons.

A partir des valeurs estimées du coefficient $K$ de Strickler et du profil en long reconstitué, nous avons calculé la courbe de remous dans l'ouvrage et éliminé les débits qui provoquent un débordement. Nous avons testé huit cas de calcul correspondant à deux valeurs de $\mathrm{K}$ et quatre valeurs du débit :

* Première hypothèse : canal non revêtu entre Zaghouan et Bou Naouara $(\mathrm{K}=30)$, canal revêtu entre Bou Naouara et La Malga $(\mathrm{K}=50)$. 
* Deuxième hypothèse : canal revêtu sur toute sa longueur $(\mathrm{K}=50)$.

Les valeurs des débits sont $18000 \mathrm{~m}^{3} / \mathrm{j}, 25000 \mathrm{~m}^{3} / \mathrm{j}$, $28000 \mathrm{~m}^{3} / \mathrm{j}$ et $32000 \mathrm{~m}^{3} / \mathrm{j}$. Cette dernière valeur provoquant des débordements avec les deux valeurs de $\mathrm{K}$, nous n'avons pas testé de débits supérieurs.

L'écoulement étant fluvial, au moins entre Moghrane et La Malga, la condition choisie à la limite aval est une hauteur critique, en supposant un déversement de l'aqueduc dans les citernes.

Dans le cas de la première hypothèse, les quatre débits de projet provoquent des débordements sur le tronçon non revêtu, au niveau des faibles pentes ou des contre-pentes (deux débordements pour le débit de $18000 \mathrm{~m}^{3} / \mathrm{j}$ ). Remarquons toutefois que la valeur 30 est une valeur minimale de $\mathrm{K}$, correspondant à un écoulement en présence de dépôts. D'autre part, le débit de $18000 \mathrm{~m}^{3} / \mathrm{j}$, actuellement imposé par la SONEDE ne provoque pas de débordements de nos jours. Le débit maximal réel de l'ouvrage est donc au moins égal à cette valeur.

Dans le cas de la deuxième hypothèse, seuls les débits de $28000 \mathrm{~m}^{3} / \mathrm{j}$ et de $32000 \mathrm{~m}^{3} / \mathrm{j}$ provoquent des débordements, voire, pour le plus grand débit, des vitesses supérieures à la vitesse admissible. La valeur 50 étant une valeur maximale de $\mathrm{K}$ sur l'aqueduc, nous pouvons considérer ces deux débits comme supérieurs au débit maximal de l'ouvrage.

La valeur $\mathrm{Q}=25000 \mathrm{~m}^{3} / \mathrm{j}$ sera donc choisie comme un ordre de grandeur raisonnable de la capacité maximale de l'aqueduc.

\section{Consommation en eau à l'époque romaine}

Il est bon, avant d'estimer la consommation en eau de Carthage au deuxième siècle, de rappeler quelques chiffres actuels: en 1988, la population tunisienne urbaine consomme $150 \mathrm{l} / \mathrm{hab} . / \mathrm{j}$. Ce chiffre descend à $40 \mathrm{l} / \mathrm{hab} . / \mathrm{j}$ dans les zones rurales desservies par des fontaines publiques. A l'étranger, cette consommation atteint $400 \mathrm{l} / \mathrm{hab}$./j dans les pays développés.

Les enquêtes archéologiques menées à Rome et Pompéï font état pour ces deux villes d'une consommation de $450 \mathrm{l} / \mathrm{hab}$./j à l'époque romaine. Ce chiffre est comparable à la valeur observée de nos jours dans les pays les plus industrialisés et paraît donc excessif. La seule explication que l'on peut avancer est que l'eau coulait en permanence dans les aqueducs, ce qui donnait lieu à un gaspillage considérable. Cette remarque est confirmée par l'existence dans les villes romaines de réseaux d'égouts fort développés.

Pour la ville de Carthage, et sur la base d'une population de 100000 hab. (Lézine, 1969), la consommation en eau de la ville à partir de l'aqueduc serait, selon nos estimations, de $250 \mathrm{l} / \mathrm{hab}$./j. Il faut rajouter à cela les consommations à partir des citernes privées ou publiques. Ces consommations sont estimées à :
- $500000 \mathrm{~m}^{3}$ annuels pour les citernes privées sur la base d'un volume unitaire des citernes de $50 \mathrm{~m}^{3}$ (Fakhar et Soussi, 1988) et d'un taux d'occupation de 10 hab./logement (Lézine, 1969);

- $500000 \mathrm{~m}^{3}$ annuels pour les citernes publiques, estimés à partir du volume des citernes de Borj Djedid et La Malga, en supposant arbitrairement que ces citernes se remplissaient deux fois dans l'année et en prenant un coefficient majorateur de 3 pour tenir compte d'éventuelles autres citernes dont les traces auraient disparu.

Bien que ces chiffres soient des valeurs maximales, ils ne correspondent qu'à un débit supplémentaire de $10 \mathrm{l} / \mathrm{hab}$./j.

La consommation en eau à Carthage au ${ }^{\mathrm{e}}$ siècle serait donc de l'ordre de 260 l/hab./j. La différence notable avec la valeur estimée à Rome s'explique alors par le haut niveau de vie de la capitale romaine.

\section{Conclusion}

Les documents historiques de l'époque romaine ou les études plus récentes montrent que les Romains avaient une bonne connaissance de l'hydraulique qui se manifestait surtout dans les ouvrages annexes des aqueducs. Cette connaissance est fondée sur une large part d'empirisme, comme en témoignent certaines incertitudes dans la définition des pentes. Ainsi, la définition des caractéristiques hydrauliques de l'ouvrage, sur la base des connaissances actuelles, nécessite donc des mesures in situ. L'étude est alors limitée par le problème des données: état de l'ouvrage, incertitude des anciens tracés, modifications subies par l'ouvrage dans le temps, agressions humaines.

Cette étude a le mérite, sur la base de travaux expérimentaux, relevés topographiques, observations et enquêtes sur terrain, d'avoir dégagé un certain nombre d'hypothèses réalistes qui ont permis de compléter les profils en long notamment dans la partie aval et estimer la rugosité de l'ouvrage. Des études plus ambitieuses, faisant intervenir des travaux topographiques, cartographiques et des fouilles sur l'ensemble des ruines, permettraient sans doute une reconstitution plus précise du tracé en long ainsi qu'une meilleure compréhension du débouché de l'aqueduc et de la répartition ultérieure de l'eau à son arrivée à Carthage. Ces études sont d'autant plus urgentes que l'urbanisation rapide des banlieues de Tunis risque de dégrader définitivement les traces du monument.

Toutefois, les résultats obtenus pour la consommation en eau de Carthage montrent que celle-ci se situe bien dans les ordres de grandeur des consommations de Rome et de Pompéï. Le chiffre plus faible trouvé pour Carthage, peut s'expliquer par le haut niveau de vie de la capitale romaine ainsi que par les habitudes différentes de consommation des deux pays.

Une voie de recherche, dans la connaissance des techniques romaines, est l'étude des réseaux d'assainissement qui, selon les chiffres trouvés dans cette étude, devaient évacuer des débits comparables à ceux de nos grandes agglomérations urbaines contemporaines. 


\section{Bibliographie}

[1] Caillat, Ph. - Extrait d'une note sur la restauration de l'ancien aqueduc de Carthage. Revue archéologique, XXVI, 1873.

[2] CHow, V. T. - Open channel hydraulics. Mac Graw-Hill, 1959.

[3] FAKHAR, R. et SousSI, A. - Recherche sur la meilleure conception et gestion des citernes en zones urbaines. Projet de fin d'études, Ecole Nationale d'Ingénieurs de Tunis. Tunis, 1988.

[4] Gafrej, R., Smaoul, L. - Hydraulique de l'aqueduc romain de Carthage. Projet de fin d'études, Ecole Nationale d'Ingénieurs de Tunis. Tunis, 1988.

[5] GauCKLer, P. - Enquêtes sur les installations hydrauliques romaines de Tunisie. Direction des Antiquités et Beaux-Arts, Tunis, vol. II, 1900.

[6] LARRAS, J. - Hydraulique et granulats. Eyrolles, 1972.

[7] Leveau, Ph. et Palllat, J. L. - L'alimentation en eau de Caesarea de Mauritanie et l'aqueduc de Cherchell. Paris, Harmattan, 1975.

[8] LeZine, A. - Sur la population des villes africaines. Extrait de: Antiquités Africaines, t. 3, Paris, Ed. du CNRS, 1969.

[9] Raков, F. - Le sanctuaire des eaux à Zaghouan. Africa III et IV, 1969-1970.

[10] RАКов, F. - Des deutschen archaelogischen instituts roemische. Albeilwg. Band 81, 1974.

[11] Rаков, F. - Aqueduc de Carthage. Dossier de l'Archéologie, $\mathrm{n}^{\circ} 38$, octobre/novembre, Paris 1979.

Cette étude a été menée à l'Ecole nationale d'ingénieurs de Tunis sur l'initiative de Monsieur Boussabah (Groupement d'Ingénieurs Conseil) avec le concours de Monsieur Slim et Mademoiselle Ferchiou de l'Institut national d'Art et d'Archéologie. Nous les remercions pour leur collaboration. 


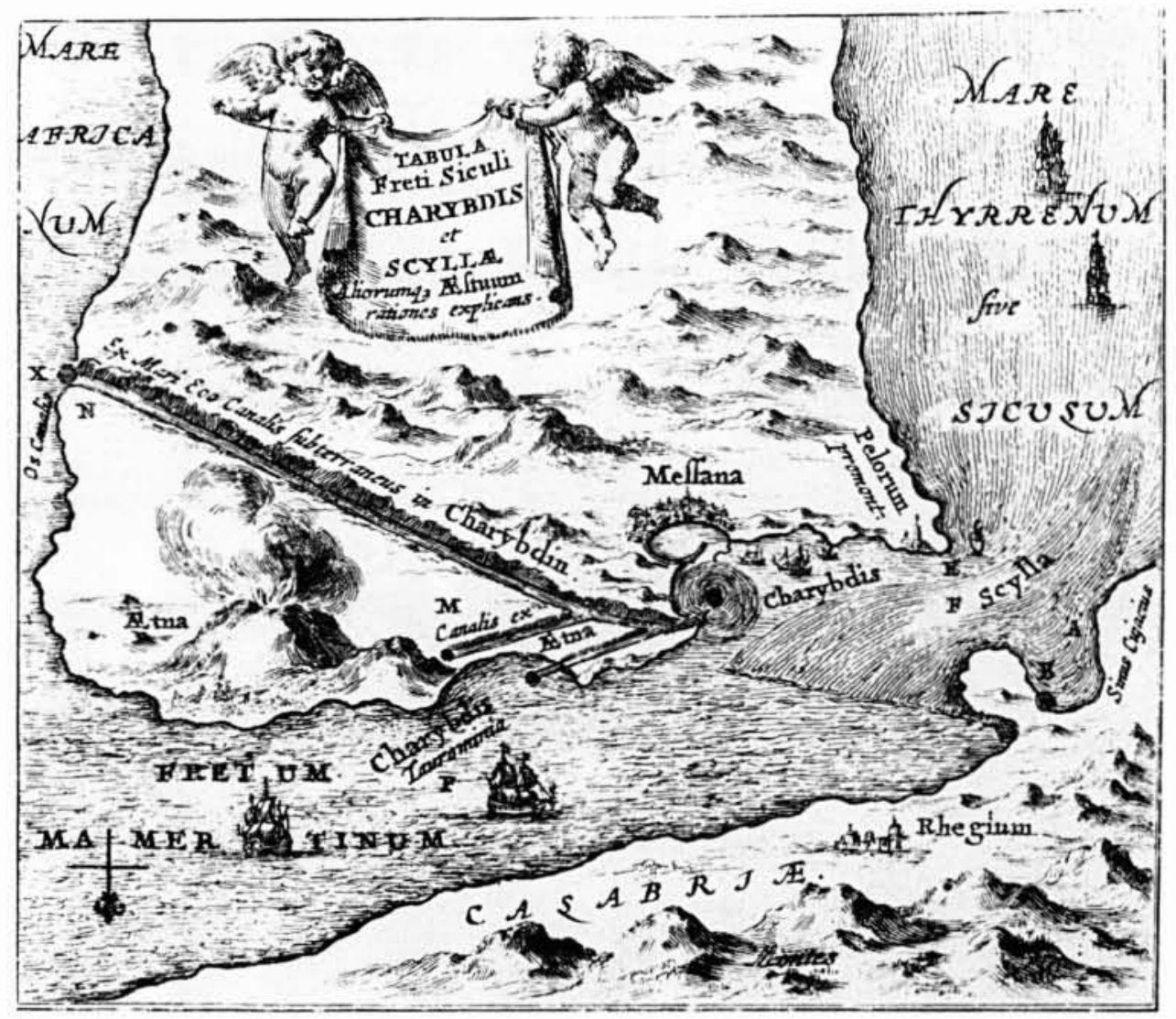

De admirandis mundi cataractis - Jean Herbinius - Amsterdam - 1678. 